\title{
Nonlinear similar design of large slenderness ratio truss boom
}

\author{
Xu-yang CAO*, Peng WANG* and Wen-jun WANG* \\ * School of Mechanical Engineering, Dalian University of Technology \\ Dalian, Liaoning, China \\ E-mail: wwj010751836@126.com
}

Received: 27 December 2016; Revised: 9 May 2017; Accepted: 23 July 2017

\begin{abstract}
To solve the problem that lacks systematic product spectrum plan and serialization of design methods and theoretical basis in the design process of crawler crane boom, the main boom is as the research object in this paper. Force model is established in luffing plane and rotary plane by the load combination II when the lifting mechanism and the rotating mechanism simultaneously works. On the premise of each demand parameters of prototyping products being known, FEM basic equations are expressed in the form of potential function by introducing displacement potential function based on the local coordinate system. Unit similarity criterion is established in the local coordinate system by non-dimensional treatment for FEM basic equations are expressed in the form of displacement potential function. Nonlinear similarity criterion in the global coordinate system is obtained by using coordinate transformation method. The chord member cross-sectional area of design goals is designed by nonlinear similarity criterion and stability principle. The width and height of the boom is calculated based on rigidity principle. The layout of web member has been discussed. The angle between web member and chord member is designed as well as the length of web member.
\end{abstract}

Key words : Crawler crane, Boom design, Nonlinearity, Similarity criterion

\section{Introduction}

The large lifting machinery boom system, which is often used in the large engineering field such as the exploitation of energy resources, has a structure of truss boom practically. The large slenderness ratio structure and material requirement of high strength with small mass bring nonlinear characteristics to this kind of structure. Stand-along design and manufacture with this structural characteristics lack systematic product spectrum planning, serialized design method and theoretical basis (Zhang, H.S, 2010).

The enlightenment of similarity between things and the use of rules of similarity are both the bridge which must be passed connected to serialized and creative design. Similarity theory is a theory which studies every kind of similarity principle of physical phenomenon in natural world or engineering field and this theory has some mature applies in model tests. It can be used to reduce design cycle of products or even make the new product more creative based on those fine mechanical properties of the product in prior version, if similarity design method be used in design process of mechanical products.

As the development of science and technology, many scholars gradually apply similarity theory to engineering field, such as the similar design of engineering system or man-machine system, similar virtual manufacture, group technology and similar design of mechanical system. In reference (Islam, K.P, 2000) and (Taesik, H,2006), group technology is applied in practical projects to help directing engineering design. Taesik Hong proposed the expressive 
method based on Brep features and a kind of similar design method which compares the similarity between local and global aspects and can be used in new design. Du Feng-shan established the similar finite element theory of elastostatics problems and theoretically proved that corresponding stress and strain of prototype and model change along with similarity criterions (Du, F.S, 1998). Lei Zheng-bao applied similarity theory to dynamic analysis of large deformation of vehicle structure by setting similarity criterions and similar computational accuracy of large deformation of vehicle structure. This created the conditions for similar design and computation of complicated structure with large deformation (Lei, Z.B, 2000).

The key purpose of classic similarity theory is to help directing model tests, which can be seen through analysis of similarity theory itself and its applications. Although similarity theory has some applications in similar design, these applications are still limited in the design of linear system. It lacks reliable theoretical analysis method for nonlinear system. Lots of scholars have made great progress in large deflection problems of traditional nonlinear engineering structure using similarity theory. But similarity theory still cannot be used to practical engineering projects synthetically because corresponding theories are sporadic relatively.

The applications in engineering design field by using similarity theory are limited for these problems of classic similarity theory when it is applied in nonlinear problems which are mentioned above. The fact that most problems in engineering field can be said belong to nonlinear problems for some degree proves the universality of this problem

Crane design enters a new stage with the development of computer and finite element numerical methods, such as optimization design, finite element design, modular design and other modern design methods. But these methods belong to a kind of 'post-design' approach, which check improvement or numerical optimization on the basis of initial structure parameters. These methods still don't solve the problems mentioned above. It is still difficult for enterprises to act rapidly according to the change of market because there's no creative design method which can meet the requirements of rapidness and serialization (Okamoto, 2007; Yuan, B, et al, 2000; Chetan, S and Michelle, V, 1996; Mike, O, 1998; Shi, M.J, 2010).

For the purpose of solving these problems mentioned above, reducing cost, increasing efficiency of design and intensifying succession of benchmarking product which has been optimized, this article deduce out nonlinear similar design method of target product based on prototype product under large deformation condition, improving the problem that classic similarity theory is limited when it's used in nonlinear problems.

\section{Force analysis and equivale nt method of moment of ine rtia of truss works 2.1 Force analysis of main boom in truss work}

The main boom that is widely used in large slenderness ratio structures is selected as the research object in this paper. In this paper, the selected working condition of the main boom is the most typical one among different combination kinds of booms of crawler crane. This kind of working condition is selected base on risk degrees of different working conditions and it allows lifting and rotating mechanis m work simultaneously. Load combination II (SAPRC, 2008) is set as the working load, which contains gravity, load under lifting, pulls of raising line and luffing line, wind load and inertia force under rotating and etc.

\subsubsection{Force analysis of truss work in luffing plane}

Booms do luffing motion around the axis pin set at the bottom under the effect of luffing line set at the head. For this reason, in luffing plane, these booms can be replaced by simple supported beams which are hinged both sides, with one side fixed and the other flexible. The simple force diagram is shown in Fig. 1. 

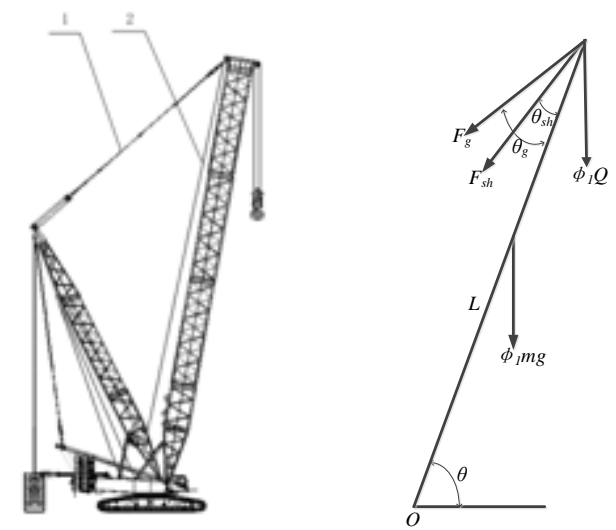

1-luffing rope 2-lifting rope

Fig. 1 Force diagram of boom in luffing plane

In luffing plane, the main loads include loads from luffing pulling plate, lifting line and lifting motion, gravity of booms and impulse load or inertial load caused by speed changes of some mechanisms.

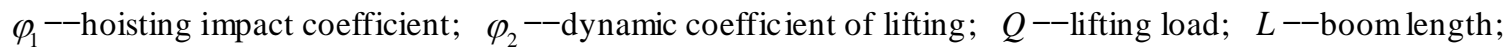

$\theta$--boom elevation; $F_{s h}$--tension of lifting rope; $F_{g}$--tension of luffing rope; $m g$--self-weight of boom;

$\theta_{s h}$--angle between lifting rope and axis of boom; $\theta_{g}$--angle between luffing rope and axis of boom;

\subsubsection{Force analys is of truss work in rotating plane}

In rotating plane, the degrees of freedom at bottom of the booms are all frozen, which equals to being fixed by hinges, and the degrees of freedom at head are no all froze. So model can be simplified as a cantilever beam under lengthways bending load, which is shown in Fig. 2.

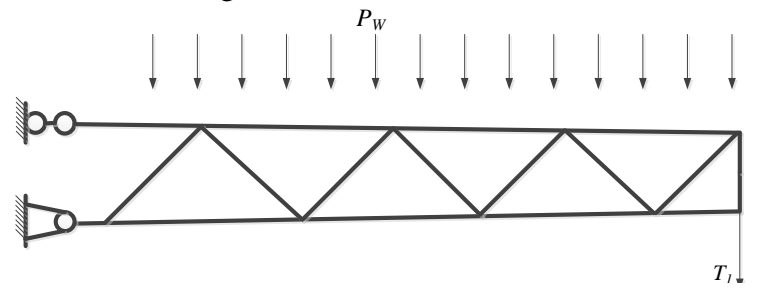

Fig. 2 Force diagram of boom in rotary plane

\subsection{Equivalent method of moment of ine rtia of truss works}

The equivalent method of moment of inertia of truss work can be used to simp lify a truss work with uniform sections or uneven sections to solid-web structure.

\subsubsection{Equivale nt method of moment of inertia of truss works with uniform sections}

The computational formu la of critical force of compression me mber in truss boom

$$
P_{c r}=\frac{\pi^{2} E I_{x}}{l_{c x}^{2}} \times \frac{1}{1+\mu_{1} \frac{\pi^{2} E I_{x}}{l_{c x}^{2}}}
$$

$P_{c r}-$-critical force of the compression bar; $E$--elasticity modulus; $I_{x}-$-moment of inertia pivoting axis $\mathrm{x}$ 


$$
I_{x}=4\left[I_{0}+A_{0} \cdot\left(\frac{H}{2}\right)^{2}\right]
$$

$A_{0}{ }^{-}$-sectional area of one main chord member; $I_{0}{ }^{--}$moment of inertia of main chord me mber $H$--height of booms; $l_{c x}$--length of component

The computational formu la of critical force of compression bar in solid-web structure.

$$
\mu_{1}=\frac{1}{2 E A_{f} \sin ^{2} \alpha \cos \alpha}
$$

$A_{f}-$-section area of web members in one side;

$\alpha$--angle between chord member and web member.

$$
P_{c r}=\frac{\pi^{2} E I_{d x}}{l_{c x}^{2}}
$$

$I_{d x}$--computational mo ment of inertia of solid-web structure

Assume that the critical force of solid-web structure is the same as that of truss work and make Eq. (1) and (2) simultaneous, and the computational formula of moment of inertia of solid-web structure can be get.

$$
I_{d x}=\frac{I_{x}}{1+\mu_{1} \frac{\pi^{2} E I_{x}}{l_{c x}^{2}}}
$$

\subsubsection{Equivalent method of moment of ine rtia of truss works with uneven sections}

Some basic assumptions of beam element with uneven sections:

(1) Beam depth changes along linear or parabola trajectories;

(2) Cross sections are symmetrical about both axis;

(3) It conforms to the beam assumption of Euler-Bernoulli in which condition deformed section is still vertical to the axis it was vertical to before deformation. Effect caused by shear deformation is ignored;

(4) All source of element is assumed to be is otropy elastic.

The computational formu las of sectional area and moment of inertia in any positions for beams with uneven sections are as follows whose depth changes along linear or parabola trajectories.

$$
\left\{\begin{array}{l}
A=A_{1}\left(1+\alpha \xi^{m}\right) \\
I=I_{1}\left(1+\beta \xi^{m}\right)^{n}
\end{array}\right.
$$

Subscripts 1 and 2-- side $\mathrm{i}$ and $\mathrm{j}$ of the element; $A_{1}-$-sectional area of side i; $I_{1}--$ mo ment of inertia of side i; $\quad \xi--\mathrm{a}$ parameter with no dimensions, the range of its value is $0 \sim 1 ; m 、 n--$ parameters related to the changing rules of beam depth. Set $m=1 、 n=3$ if beam depth changes along linear trajectory and set $m=2 、 n=3$ if along parabola trajectory.

$$
\left\{\begin{array}{l}
\alpha=\frac{A_{2}}{A_{1}}-1 \\
\beta=\sqrt[3]{\frac{I_{2}}{I_{1}}}-1
\end{array}\right.
$$

$\alpha--$ coefficient related to the changes of sectional area; $\beta$--coefficient re lated to the changes of mo ment of inertia.

\section{The method to derive nonlinear similarity crite rions based on similarity theory}


Linear similarity theory can be applied in element coordinate system to solve nonlinear problems under these assumptions as follows:

(1) Large deformations of elements equal superposition of s mall elastic deformation in element coordinate s ystem or relative ele ment coordinate system;

(2) equilibrium equations are established on the conditions after deformation and through these equations, the orientation matrix can be derived;

(3) local deformations of element is relatively s mall, so elastic theory for s mall deformations can be used here.

Based on the ideology of method of establishing similarity criterions in reference (Cheng, H.Z, 2013), it's assumed under constant temperature and the effect of temperature field is ignored. The linear similarity criterions in element coordinate system are derived first and nonlinear similarity criterions are gained by transforming similar relationships in ele ment coordinate system to global coordinate system.

\subsection{The process of deducing the similarity crite rions under element coordinate system 3.1.1 Finite element me thod basic equations represented by potential function}

Physical equation, geo metric equation, equilibriu m equation, boundary condition of stress and boundary condition of strain are as follows:

$$
\begin{gathered}
\varepsilon=C^{-1} \sigma \\
\varepsilon=L U \\
L^{T} \sigma+\bar{f}=0 \\
\bar{P}=n \sigma+\bar{K} U \\
U=\bar{U}
\end{gathered}
$$

$\varepsilon$--array of strain; $\sigma-$-array of stress; $L--$ matrix of operators in partial differential equations;

$U$--array of displacement in directions $\mathrm{x}, \mathrm{y}, \mathrm{z} ; \bar{f}$--array of internal forces distributed in directions $\mathrm{x}, \mathrm{y}, \mathrm{z}$;

$\bar{P}$--array of boundary stress; $\bar{K}$--matrix of elastic restraint constants on boundaries;

$n$--direction cosine matrix in direction of outer normal of boundaries; $C$--matrix of rigidity.

Substitute Eq. (5) and (6) into (7) and (8) and then some new equations can be get:

$$
\begin{gathered}
L^{T} C L U+\bar{f}=0 \\
\bar{P}=n C L U+\bar{K} U
\end{gathered}
$$

Introduce displacement potential function $\varphi(x, y, z)$ in and define it in the relationship with displacement quantities as follows:

$$
u=\frac{\partial \varphi}{\partial x}, v=\frac{\partial \varphi}{\partial y}, w=\frac{\partial \varphi}{\partial z}
$$

Express these equations in form of matrix:

$$
U=A \varphi
$$

$A-$ matrix of operators in partial differential equations.

Substitute Eq. (12) into (9), (10) and (11) respectively and get three new equations:

$$
\begin{gathered}
L^{T} C L A \varphi+\bar{f}=0 \\
\bar{P}=n C L A \varphi+\bar{K} A \varphi \\
A \varphi=\bar{U}
\end{gathered}
$$

\subsubsection{Non-dimensional tre atment of basic equations expressed by potential function}


After introducing scale parameters and making them through non-dimensional treatment, if each parameter with no dimensions is substituted into Eq. (13) and (15), the new equations without dimensions will be derived (only use the equilibriu $\mathrm{m}$ equation in direction $\mathrm{X}$ as an example).

Equilibrium equation with no dimensions in direction $\mathrm{X}$ is as follows:

$$
\begin{aligned}
& \frac{c_{11}}{f_{x 0}} \frac{\varphi_{0}}{A^{3}} \frac{\partial^{3} \varnothing}{\partial X^{3}}+\frac{c_{12}+2 c_{66}}{f_{x 0}} \frac{\varphi_{0}}{A B^{2}} \frac{\partial^{3} \varnothing}{\partial x \partial Y^{2}}+\frac{c_{26}}{f_{x 0}} \frac{\varphi_{0}}{B^{3}} \frac{\partial^{3} \varnothing}{\partial Y^{3}}+\frac{2\left(c_{14}+2 c_{56}\right)}{f_{x 0}} \frac{\varphi_{0}}{A B C} \frac{\partial^{3} \varnothing}{\partial X \partial Y \partial Z}+\frac{c_{13}+2 c_{55}}{f_{x 0}} \frac{\varphi_{0}}{A C^{2}} \frac{\partial^{3} \varnothing}{\partial X \partial Z^{2}}+ \\
& \frac{c_{35}}{f_{x 0}} \frac{\varphi_{0}}{C^{3}} \frac{\partial^{3} \varnothing}{\partial Z^{3}}+\frac{3 c_{16}}{f_{x 0}} \frac{\varphi_{0}}{A^{2} B} \frac{\partial^{3} \varnothing}{\partial X^{2} \partial Y}+\frac{3 c_{15}}{f_{x 0}} \frac{\varphi_{0}}{A^{2} C} \frac{\partial^{3} \varnothing}{\partial X^{2} \partial Z}+\frac{c_{25}+2 c_{46}}{f_{x 0}} \frac{\varphi_{0}}{B^{2} C} \frac{\partial^{3} \varnothing}{\partial Y^{2} \partial Z}+\frac{2 c_{45}+c_{36}}{f_{x 0}} \frac{\varphi_{0}}{B C^{2}} \frac{\partial^{3} \varnothing}{\partial Y \partial Z^{2}}+F_{x}=0
\end{aligned}
$$

\subsubsection{The me thod to derive similarity crite rions and relations hip between them based on non-dimensional equations}

From those non-dimensional equations in 3.1.2, it can be proved that the displacement potential function with no dimensions $\phi$ is function of every non-dimensional coefficients including $\mathrm{X}, \mathrm{Y}, \mathrm{Z}, F_{x}, F_{y}, F_{z}, p_{x}, p_{y}, p_{z}$, $\bar{U}, \bar{V}, \bar{W}$. In order to equalize the displacement potential functions of prototype and model, the non-dimensional coefficients of prototype and model must be equal, which means similarity criterions equal with each other correspondingly.

The similarity criterions obtained from non-dimensional equations are shown in Tab. 1.

Table 1 Similarity criterion

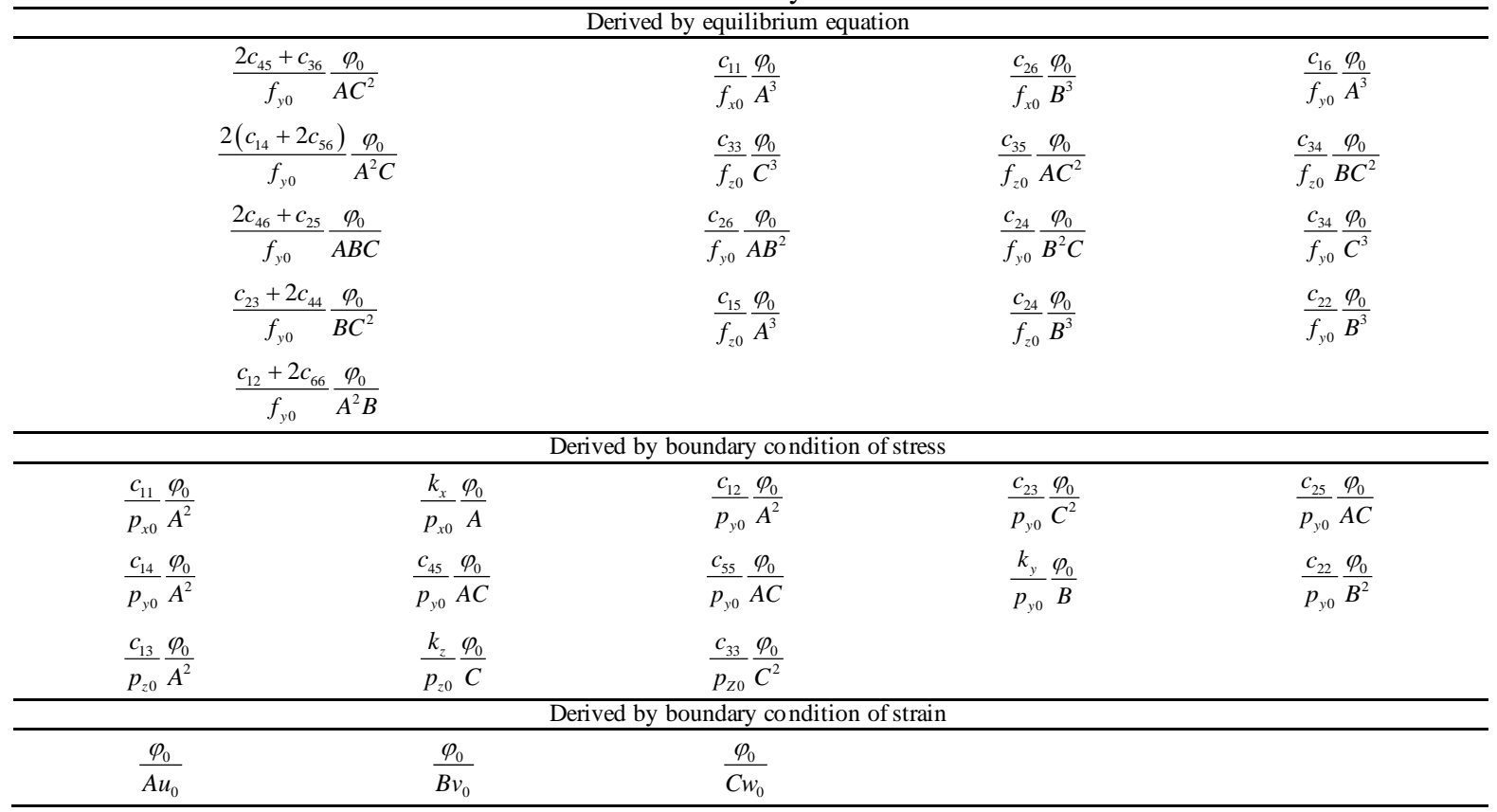

Magnify one directional size for $\mathrm{N}$ times, for example the size in direction $\mathrm{X}$, and make non-dimensional potential function of element in prototype equals that in model.

$$
\varnothing_{\mathrm{p}}(\mathrm{X}, \mathrm{Y}, \mathrm{Z})=\varnothing_{\mathrm{m}}(\mathrm{X}, \mathrm{Y}, \mathrm{Z})
$$

That is to say, displacement potential function does not change through magnification of one directional size.

$$
\varphi_{p}(x, y, z)=\varphi_{m}(N x, y, z)
$$

$p$--element in the prototype before size magnification; $m$--element in model after size magnification.

The parameters in element of prototype and model are in the similar relationship according to the similarity criterion 
shown in Tab.1.

The similar relationship between matrixes of rigidity:

$$
\left[\begin{array}{llllll}
c_{11} & c_{12} & c_{13} & c_{14} & c_{15} & c_{16} \\
c_{12} & c_{22} & c_{23} & c_{24} & c_{25} & c_{26} \\
c_{13} & c_{23} & c_{33} & c_{34} & c_{35} & c_{36} \\
c_{14} & c_{24} & c_{34} & c_{44} & c_{45} & c_{46} \\
c_{15} & c_{25} & c_{35} & c_{45} & c_{55} & c_{56} \\
c_{16} & c_{26} & c_{36} & c_{46} & c_{56} & c_{66}
\end{array}\right]_{m} \quad\left[\begin{array}{cccccc}
N^{4} c_{11} & N^{2} c_{12} & N^{2} c_{13} & N^{2} c_{14} & N^{3} c_{15} & N^{3} c_{16} \\
N^{2} c_{12} & c_{22} & c_{23} & c_{24} & N c_{25} & N c_{26} \\
N^{2} c_{13} & c_{23} & c_{33} & c_{34} & N c_{35} & N c_{36} \\
N^{2} c_{14} & c_{24} & c_{34} & c_{44} & N c_{45} & N c_{46} \\
N^{3} c_{15} & N c_{25} & N c_{35} & N c_{45} & N c_{55} & N^{2} c_{56} \\
N^{3} c_{16} & N c_{26} & N c_{36} & N c_{46} & N^{2} c_{56} & N^{2} c_{66}
\end{array}\right]_{p}
$$

The relationship between internal forces distributed in different directions:

$$
\left\{\begin{array}{l}
f_{x m}(N x, y, z)=N f_{x p}(x, y, z) \\
f_{y m}(N x, y, z)=f_{y p}(x, y, z) \\
f_{z m}(N x, y, z)=f_{z p}(x, y, z)
\end{array}\right.
$$

The relationship between boundary stresses:

$$
\left\{\begin{array}{c}
p_{x m}(N x, y, z)=N^{2} p_{x p}(x, y, z) \\
p_{y m}(N x, y, z)=p_{y p}(x, y, z) \\
p_{z m}(N x, y, z)=p_{z p}(x, y, z) \\
k_{x m}=N^{3} k_{x p}, k_{y m}=k_{y p}, k_{z m}=k_{z p}
\end{array}\right.
$$

The relationship between boundary dis placements:

$$
\left\{\begin{array}{c}
\bar{u}_{m}(N x, y, z)=N^{-1} \bar{u}_{P}(x, y, z) \\
\bar{v}_{m}(N x, y, z)=\bar{v}_{P}(x, y, z) \\
\bar{w}_{m}(N x, y, z)=\bar{w}_{P}(x, y, z)
\end{array}\right.
$$

The potential functions of prototype and model equal with each other if all the equations above are tenable.

The similar relationship between components of displacement, stress and strain can be derived by expressing physical equation and geometric equation with no dimensions.

The similar relationship between displacement components:

$$
\left\{\begin{array}{c}
u_{m}(N x, y, z)=N^{-1} u_{p}(x, y, z) \\
v_{m}(N x, y, z)=v_{p}(x, y, z) \\
w_{m}(N x, y, z)=w_{p}(x, y, z)
\end{array}\right.
$$

The similar relationship between stress components:

$$
\left\{\begin{array}{c}
\sigma_{x m}(N x, y, z)=N^{2} \sigma_{x p}(x, y, z) \\
\sigma_{y m}(N x, y, z)=\sigma_{y p}(x, y, z) \\
\sigma_{z m}(N x, y, z)=\sigma_{z p}(x, y, z) \\
\tau_{x y m}(N x, y, z)=N \tau_{x y p}(x, y, z) \\
\tau_{y z m}(N x, y, z)=\tau_{y z p}(x, y, z) \\
\tau_{x z m}(N x, y, z)=N \tau_{x z p}(x, y, z)
\end{array}\right.
$$

The similar relationship between strain components:

$$
\left\{\begin{array}{c}
\varepsilon_{x m}(N x, y, z)=N^{-2} \varepsilon_{x p}(x, y, z) \\
\varepsilon_{y m}(N x, y, z)=\varepsilon_{y p}(x, y, z) \\
\varepsilon_{z m}(N x, y, z)=\varepsilon_{z p}(x, y, z) \\
\gamma_{x y m}(N x, y, z)=N^{-1} \tau_{x y p}(x, y, z) \\
\gamma_{y z m}(N x, y, z)=\tau_{y z p}(x, y, z) \\
\gamma_{x z m}(N x, y, z)=N^{-1} \tau_{x z p}(x, y, z)
\end{array}\right.
$$

\subsection{Parame tric s witch from element coordinate system to global coordinate system}


The method of deriving out these similarity criterions in section 3.1 is based on theory of small elastic deformation in element coordinate system. It's necessary to transform the computational variables from element coordinate system to global coordinate system for the purpose of getting similar relationships in global coordinate system.

As shown in Fig. 3, the element coordinate system in the form of simply supported beams is taken as an example. In this coordinate system, one side of the element is set as the original point and the center shaft of the element is set as the $x$ axis (Wang, H.H, et al, 1997a, 1997b; Dong, S.L, et al, 2002; Lu, N.L, et al, 1998; Sun, H.C, et al, 2009).

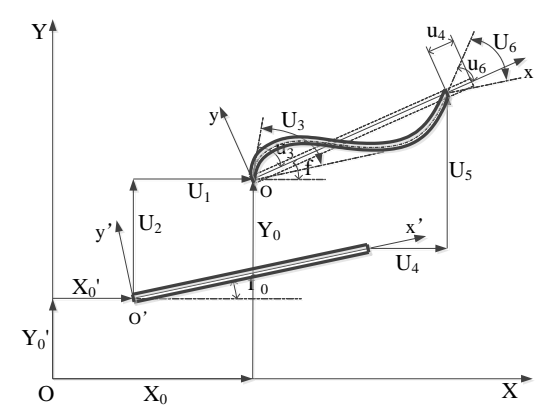

Fig. 3 The hinged-hin ged moving coordinate svstem

It can be derived from geometric relationships:

$$
u=T U+U_{0}
$$

$u=\left\{u_{1}, u_{2}, u_{3}, u_{4}, u_{5}, u_{6}\right\}^{T} \quad u$--vector quantity of displacements of nodes in element coordinate system

$U=\left\{U_{1}, U_{2}, U_{3}, U_{4}, U_{5}, U_{6}\right\}^{T} U$--vector quantity of displacement of nodes in global coord inate system

$$
T=\left[\begin{array}{cccccc}
0 & 0 & 0 & 0 & 0 & 0 \\
0 & 0 & 0 & 0 & 0 & 0 \\
0 & 0 & 1 & 0 & 0 & 0 \\
-c & -s & 0 & c & s & 0 \\
0 & 0 & 0 & 0 & 0 & 0 \\
0 & 0 & 0 & 0 & 0 & 1
\end{array}\right] \quad c=\cos \theta \quad s=\sin \theta
$$

$\theta$--the angle between current ele ment coordinate system and global coord inate system; $U_{0}--$ vector quantity of extra displacement.

$$
U_{0}=\left\{\begin{array}{c}
0 \\
0 \\
-\theta+\theta_{0} \\
-L\left(1-\cos \cos \left(\theta-\theta_{0}\right)\right) \\
0 \\
-\theta+\theta_{0}
\end{array}\right\}
$$

$\theta_{0}$--the angle between in itial element coordinate system and global coordinate system.

The following equation can be derived from $u_{s}=0$

$$
s\left(U_{1}-U_{4}-L c_{0}\right)+c\left(U_{5}-U_{2}+L s_{0}\right)=0
$$

The following equation is the explicit formu lation of angle

$$
\varphi=\arctan \left(\frac{U_{5}-U_{2}+L \sin \sin \theta_{0}}{U_{4}-U_{1}+L \cos \cos \theta_{0}}\right)
$$




\section{The similar design method of the truss work with large slenderness ratio}

The similar design of booms is based on the as sumption that the lengths of booms are known.

For the reason that the targeted product has requirements of higher lifting altitude and larger angle of range than prototype product, the length of model to prototype ratio is set as basic enlargement factor.

$$
n=L_{m} / L_{p}
$$

$n$--enlarge factor of the lengths of booms $L_{m}$--the length of the targeted boom $L_{n}$--the length of prototype boom

The targeted element can be derived by magnify ing the prototype element for $n$ times based on the method of similar relationships between elements introduced in section 3.1. The similar design of the whole cantilever crane is after transforming element parameters to global coordinate system through transform matrix of coordinate.

It is assumed that all parameters of prototype product are already known and represent it by subscript $\mathrm{m}$ when designing this model.

\subsection{Similarity design of section of the chord member in truss work}

Ord inarily, the most dangerous section in truss work is among the following sections: the position in the top boom or the bottle boom where it connects to reinforcing plates and the position in the middle of the truss work. One more section is needed where the size of the main chord member changes. The position in the middle of the truss work is selected to pick necessary parameters because the purpose of this paper is to design section area of the chord member of standard section in truss work.

The similar design work of section area of standard section in truss work is carried out after picking necessary parameters from the position in the middle of the truss work. It has been defined that $p_{c r p}^{\prime}$ represents axial load of element of prototype product in local coord inate system and $\sigma_{c r p}^{\prime}$ represents critical force in local coordinate system.

The similar relationships of boundary force and component of stress derived from section 3.1 are as follows.

$$
\left\{\begin{aligned}
p_{c r m}^{\prime} & =n^{2} p_{c r p}^{\prime} \\
\sigma_{c r m}^{\prime} & =n^{2} \sigma_{c r p}^{\prime} \\
k_{x m}^{\prime} & =n^{3} k_{x p}^{\prime}
\end{aligned}\right.
$$

$p_{c r m}^{\prime}--$ critical load of design product on the boundary in element coordinate system

$\sigma_{c r m}^{\prime}--$ critical stress of design product in ele ment coordinate system

$k_{r m}^{\prime}$--elastic restraint constant on the boundary

The critical loads on the boundary of required product and prototype product $T_{p r m}$ and $T_{p m}$ can be derived from the method introduced in section 3.2. $T_{\sigma m}$ and $T_{\sigma p}$ are represent the transform matrixes of critical stress. If all parameters are transformed from element coordinate system to global coordinate system, the equations of critical load on boundary and critical stress are as follows.

$$
\left\{\begin{aligned}
p_{c r m} & =T_{p m} p_{c r m}^{\prime} \\
\sigma_{c r m} & =T_{\sigma m} \sigma_{c r m}^{\prime} \\
p_{c r p} & =T_{p p} p_{c r p}^{\prime} \\
\sigma_{c r p} & =T_{\sigma p} \sigma_{c r p}^{\prime}
\end{aligned}\right.
$$

$p_{c r m}-$-critical load on boundary of design product in global coordinate system

$\sigma_{c r m}--$ critical stress of design product in global coordinate system

$p_{c r p}--$ critical load on boundary of prototype product in global coordinate system 
$\sigma_{c r p}-$-critical stress of prototype product in global coordinate system

$T_{p m}$--transfer matrix of design product axial force; $T_{p m}$--transfer matrix of prototype axial force

$T_{p m}$--transfer matrix of design product critical stress; $T_{p m}-$-transfer matrix of prototype critical stress

The following equations are derived by pre-multiplying corresponding transform matrixes respectively.

$$
\left\{\begin{array}{l}
p_{c r p}^{\prime}=T_{p p}^{T} p_{c r p} \\
\sigma_{c r p}^{\prime}=T_{\sigma p}^{T} \sigma_{c r p}
\end{array}\right.
$$

Make Eq. (26) and Eq. (27) simultaneous, and a new equation can be gained as follows.

$$
A_{m}=\frac{p_{c r m}}{\sigma_{c r m}}=\frac{T_{p m} p_{c r m}^{\prime}}{T_{\sigma m} \sigma_{c r m}^{\prime}}=\frac{T_{p m}}{T_{\sigma m}} \frac{T_{p p}^{T}}{T_{\sigma p}^{T}} A_{P}
$$

$A_{m}$--the section area of chord members of design product

$A_{p}$--the section area of chord members of prototype product

The relationship between sections of booms of required product and prototype product can be derived from the above equation. As a result, the section area of required product can be got based on that area of prototype product.

$$
A_{m}=4 A_{0}
$$

$A_{0}$--the section area of a single chord member in required product

The section area of a single chord member can be derived by the above equation. The figure 4 shows that the $A_{m}$ is four times for $A_{0}$

The model number of main chord member in standard section can be gained by checking section area of a single chord member in corresponding tables. When it happens to those at the root of the main chord member, it's proper to select the same type or one size up kind of chord member in standard section because it has to bear larger bending moment. When it happens to those at the top position, for less bending moment, it's proper to choose one size down type of chord member to reduce the weight of booms.

\subsection{The similarity design of width and height in truss work}

It is based on the premise of meeting the whole stability of booms to design section area of the main chord member. So the design of width and height of booms should be based on the premise of the whole rigidity been satisfied.

The computational equation of rig idity in the design rules for cranes is as follows.

$$
\lambda \leq[\lambda]
$$

$\lambda--$ slenderness ratio of component

$[\lambda]$--the tolerable slenderness ratio of component by checking Standardization Ad min ist- -ration of the People 's Republic of China, 2008

The computer equations of slenderness ratio of solid-web components which carry axial loads are as follows

$$
\left\{\begin{array}{l}
\lambda_{x}=l_{c x} / r_{x} \\
\lambda_{y}=l_{c y} / r_{y}
\end{array}\right.
$$

$\lambda_{x}, \lambda_{y}-$-the computational slenderness ratios of solid-web components pivoting on axis $\mathrm{x}$ and axis $\mathrm{y}$

$l_{c x}, l_{c y}-$ the computational lengths of components pivoting on axis $\mathrm{x}$ and axis $\mathrm{y}$

$r_{x}, r_{y}$--the rotating radiuses of gross sections of components pivoting on axis $\mathrm{x}$ and axis $\mathrm{y}$

$$
r_{x}=\sqrt{I_{x} / A}, r_{y}=\sqrt{I_{y} / A}
$$

$I_{x}, I_{y}$--the moments of inertia of gross sections of the component pivoting on axis $\mathrm{x}$ and axis $\mathrm{y}$ 


$$
\left\{\begin{array}{l}
I_{x}=4\left[I_{0}+A_{0}(H / 2)^{2}\right] \\
I_{y}=4\left[I_{0}+A_{0}(B / 2)^{2}\right]
\end{array}\right.
$$

$I_{o}$--the moment of the main chord member pivoting on centre of form $H^{--}$the height of booms $B$--the width of booms $A$--the gross section area of the component

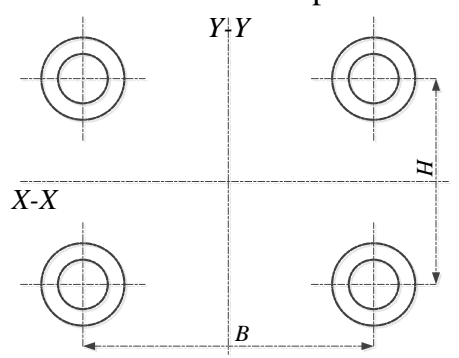

Fig. 4 Schematic diagram of boom section

The compute equations of slenderness ratios of components with equal intercepts are as follow.

$$
\left\{\begin{array}{l}
\lambda_{h x}=\sqrt{\lambda_{x}^{2}+40 A / A_{1 x}} \\
\lambda_{h y}=\sqrt{\lambda_{y}^{2}+40 A / A_{1 y}}
\end{array}\right.
$$

$\lambda_{h x}, \lambda_{h y}$--conversional slenderness ratio of latticed components pivoting on axis $\mathrm{x}$ and axis $\mathrm{y}$

$A_{1 x}, A_{1 y}$--the sums of all grass areas of all oblique lacing bars of components whose sections are vertical to x-x plane or y-y plane

New equations can be got by substituting Eq. (32) and (33) into (31).

$$
\left\{\begin{array}{l}
H \geq 2 \cdot \sqrt{\frac{l_{c x}^{2} \cdot A_{1 x}}{A_{1 x}[\lambda]^{2}-40 A}-\frac{I_{0}}{A_{0}}} \\
B \geq 2 \cdot \sqrt{\frac{l_{c y}^{2} \cdot A_{1 y}}{A_{1 y}[\lambda]^{2}-40 A}-\frac{I_{0}}{A_{0}}}
\end{array}\right.
$$

The above two equations can be used to get widths and lengths of booms.

\subsection{Similarity design of sized and layouts of web me mbers in truss work 4.3.1 The method to select the layout type of web members in truss work}

There are three main kinds of forms of web members of cranes. They are K-type structure, W-type structure and double W-type structure, which are shown in Fig. 5. K-type structure is mainly for crawler cranes of small tonnages requirement while $\mathrm{W}$-type structure is often for crawler cranes of large tonnages requirement. $\mathrm{K}$-type structure is the main form of contemporary crawler cranes because it can save weights under the required loads premises. This kind of structure has a good ability to bear vertical loads.
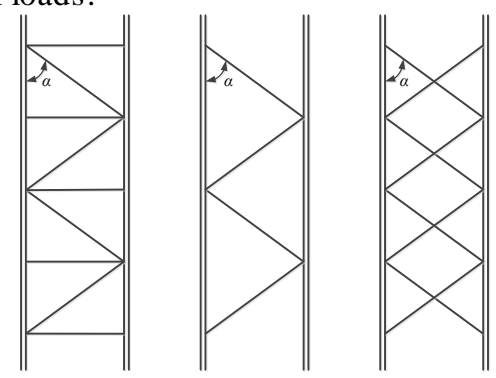

(a) K-type (b) W-type (c) double W-type 
Fig. 5 Structure diagram of web member

It often takes the triangle form of web me mbers in standard sections for its reasonable distribution. This kind of web members' distribution is symmetric around the middle section in a single web member and is anti-symmetric in re lative web members. There are three different kinds of web members, including straight type, oblique type and spatial type, whose functions and positions are shown in Tab.2. The angle between oblique web members and chord members is usually set from $55^{\circ}$ to $70^{\circ}$ to ensure the stability of web members and chord members. There two kinds of weld can be used in web members: point-to-point type and crossed type. The former type is for products of small load requirement and the latter is for those of large load requirement.

Table 2 Classification and characteristics of web member

\begin{tabular}{ccc}
\hline Type & location & function \\
\hline straight type & top & Ensure the spatial stiff \\
Oblique type & middle & Afford shear \\
Spatial type & top & Ensure the spatial stiff \\
\hline
\end{tabular}

Above all, it should take the W-type structure with crossed weld method for web members in crawler cranes with large load requirement.

\subsubsection{The method to set the angles and spans between web members}

Fig. 6 shows the similar design for the W-type structure with crossed weld.

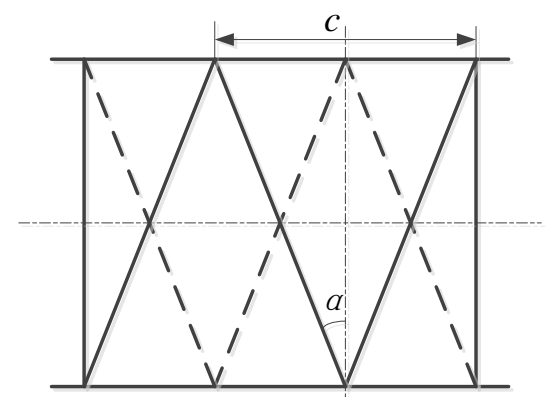

Fig. 6 Schematic diagram of single limb span

The dimensional limit should be taken into consideration when setting the lengths of booms. The length of the standard sections should be moderated. If it is too short, the weight of the boom will be higher and it need more time to be installed. Contrarily, if the boom is too long, it will enlarge the span of the lifting performance. It usually uses three types of lengths in crawler cranes with large load requirement: $3 \mathrm{~m}, 6 \mathrm{~m}$ and $12 \mathrm{~m}$. Concerning the dimensional limit of length of standard sections, it cannot use the magnification times of the whole length of booms as $\mathrm{n}$ in the similar design of standard sections. Instead, the value of $n$ should be set based on the magnification times of the standard sections under the limitation of the lengths of the standard sections.

The span of designed product can be derived based on the span of prototype product which is already known.

$$
c_{m}=n^{\prime} \cdot c_{p}
$$

$c_{m}$--the span of a single boom of designed product $\quad c_{p}$--the span of a single boom of prototype product

The equations of the angles between aimed web members and chord members are as follows

$$
\left\{\begin{array}{l}
\alpha_{H}=90^{\circ}-\arctan \frac{c_{m}}{2 H}=90^{\circ}-\arctan \frac{n^{\prime} \cdot c_{p}}{2 H} \\
\alpha_{B}=90^{\circ}-\arctan \frac{c_{m}}{2 B}=90^{\circ}-\arctan \frac{n^{\prime} \cdot c_{p}}{2 B}
\end{array}\right.
$$

$\alpha_{H}$--the angle between web member and chord member in luffing plane 
$\alpha_{B}$--the angle between web me mber and chord member in rotating plane

So the equations of the lengths of web member in luffing plane and rotating plane are as follows.

$$
\left\{\begin{array}{l}
l_{H}=H / \cos \left(90^{\circ}-\alpha_{H}\right) \\
l_{B}=B / \cos \left(90^{\circ}-\alpha_{B}\right)
\end{array}\right.
$$

$l_{H}$--the length of web me mber in luffing plane $\quad l_{B}$--the length of chord me mber in rotating plane

\section{Design instance}

Based on the Nonlinear similarity criterion established in second part and the similarity parameter design method of truss boom established in third part, the paper has made a similar design for the boom structural parameter of crawler crane, of which the rated lifting weight is 1350tonnes, by taking the QUY750 as the prototype as shown in figure 1.

\subsection{Finite element modeling of prototype}

\subsubsection{Equivalent moment of inertia of truss boom}

The boom parameters of prototype products QUY750 are shown in Tab. 3

Table 3 Boom parameters of QUY750 crawler crane

\begin{tabular}{|c|c|c|c|c|}
\hline & Standard section & Bottom section & Top section & Pendant rod section \\
\hline lengh $(\mathrm{mm})$ & 6000,12000 & 11000 & 12000 & 81800 \\
\hline $\mathrm{W} \times \mathrm{H} \quad(\mathrm{mm})$ & $2800 \times 2600$ & $2800 \times 520$ & $2400 \times 2100$ & $65 \times 36$ \\
\hline $\begin{array}{l}\text { D of chord member } \times \text { thickness } \\
(\mathrm{mm})\end{array}$ & & $\varnothing 219.1 \times 20$ & & - \\
\hline $\begin{array}{l}\text { D of web member } \times \text { thickness } \\
(\mathrm{mm})\end{array}$ & $\begin{array}{r}\varnothing 114.3 \times 6.3 \\
\text { (rotary plane) }\end{array}$ & & $\begin{array}{c}\varnothing 114.3 \times 5 \\
\text { (luffing plane) }\end{array}$ & - \\
\hline angle & $\begin{array}{c}66^{\circ} \\
\text { (rotary plane) }\end{array}$ & & $\begin{array}{c}64^{\circ} \\
\text { (luffing plane) }\end{array}$ & - \\
\hline
\end{tabular}

On basis of the equivalent method for the moment of inertia of constant section and variable section in the first part, the equivalent moments of inertia of QUY750 boom sections are shown in Tab. 4.

\begin{tabular}{cccccc}
\multicolumn{6}{c}{ Table 4 Equiv alent moment of inertia of boom sections } \\
\hline Axis X $\left(\mathrm{mm}^{4}\right)$ & $I_{1}$ & $I_{2}$ & $I_{3}$ & $I_{4}$ & $I_{5}$ \\
Axis Y $\left(\mathrm{mm}^{4}\right)$ & $9.6 \mathrm{E}+9$ & $2.6 \mathrm{E}+10$ & $7.7 \mathrm{E}+10$ & $6.4 \mathrm{E}+10$ & $5.2 \mathrm{E}+10$ \\
\hline
\end{tabular}

The location of equivalent moment of inertia in Tab. 4 can be displayed on Fig. 7 intuitively.

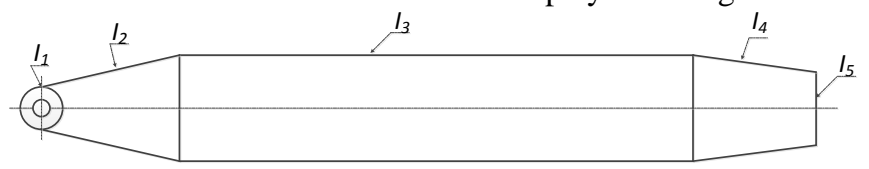

Fig. 7 Diagram of the equivalent moment of inertia

\subsubsection{Equivalent finite element model establis hment of prototype}

While the equivalent finite element model of prototype has been established, the DOFs (UX, UY, UZ, ROTX, ROTY) of boom bottom pivot point are all constrained except the DOF of rotation arou nd axis Z. Also the trans lation DOFs (UX, UY, UZ) in three directions of the luffing pendant rod bottom pivot point are constrained. The top of boom is linked with the top of luffing pendant rod so that the DOFs of its connection point are all constrained (UX, UY, UZ, ROTX, ROTY) excluding the DOF of rotation around axis Z.

The finite element model is applied with lifting load, lateral load and gravitational load. The adoption of finite element unit of boom is beam44, while the luffing pendant rod is link8.

The paper selects the operating condition that the length of main boom is $96 \mathrm{~m}$, working radius is $14 \mathrm{~m}$. In this 
working condition, the rated load weight is 227.1 tonnes. The finite element model which is simplified is shown as figure 8 .

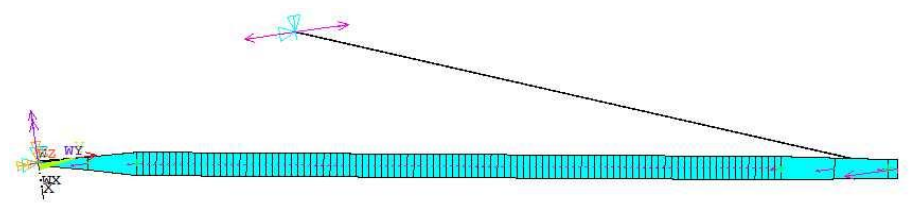

Fig. 8 Finite element model of boom

\subsection{The bucking analys is of prototype truss boom 5.2.1 Eigenvalue buckling analysis of prototype truss boom}

The linear buckling analysis which is simple to be set up and solved can be not only used to evaluate the upper limit of buckling load preliminarily but to acquire the deformation feature of the main boom. It is conducive to advanced analysis and the accuracy verification of model. The third order eigenvalues are shown in Tab. 5 by taking the bucking analysis of prototype finite element model.

Table 5 The first three eigenvalues of finite element model

\begin{tabular}{cccc}
\hline & First order & Second order & Third order \\
\hline eigenvalues & 1.0034 & 1.8466 & 4.8298 \\
\hline
\end{tabular}

The value which is the product of eigenvalues in table 5 and the load applied in boomequals to the critical buckling load.

$$
p_{t l}=\varphi_{l} \cdot F=5599400 N
$$

\subsubsection{Nonlinear analysis of prototype truss boom}

The linear buckling analysis is not able to take the initial defect and nonlinear feature of boom structure into consideration, which leads that the result of linear analysis cannot live up to the engineering application. In order to simu late the actual features of engineering structure more precisely, the paper uses the nonlinear buckling analysis.

A nonlinear buckling analysis of prototype was suggested in this paper by taking the linear buck ling load in last part as the load applied in nonlinear analysis, which can extract top node of truss boom. The load-displacement curve is shown in figure 9 through that is composed by taking the top node as observation spot.

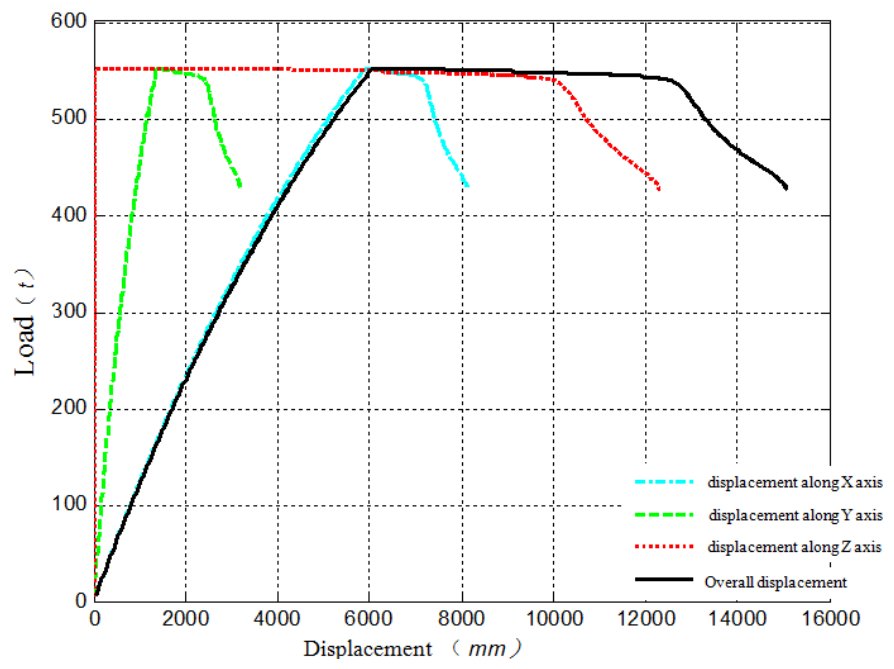

Fig. 9 Load displacement curve

The load-step in the inflection point of load displacement curve is selected as the picked point for prototype. 


\subsection{The similar design of truss boom of design objective}

The designed length of 1350tonnes crawler crane main boom is 150 meters. Its operating angle is identical to the angle of prototype QUY750, which leads the working radius equals to 25 meters.

\subsubsection{The similar design of cross-sectional area of chord member}

Read the date of prototype QUY750 boom in critical state. The node displacement is extracted by use of no. 62 unit that is the finite element in the dangerous section, which is shown in Tab. 6.

Table 6 Nodal displacement values of 62 element

\begin{tabular}{ccccccc}
\hline \multirow{2}{*}{ Node } & \multicolumn{1}{c}{$\mathrm{I}$} & $\mathrm{Y}$ & $\mathrm{Z}$ & $\mathrm{X}$ & $\mathrm{Y}$ & $\mathrm{Z}$ \\
\hline \multirow{2}{*}{ Displacement } & 3416 & -865.1 & 72.07 & 3450. & -872.1 & 73.83 \\
& .6 & 7 & 5 & 8 & 8 & 5 \\
\hline
\end{tabular}

The deformation of boomeffected by gravity is negligible in in itial state. So the angle in elevation of main boon can be assumed to equal to the angle which is between the local coordinate system and global coordinate system approximately. It can be concluded that the simplified finite element model of QUY750 boom has the initial angle of 81.6 degrees $\varphi_{0 p} \approx 81.6^{\circ}$. By the same token, the simplified finite element model of 1350tonnes crawler crane boom has the initial angle of 80.4 degrees $\varphi_{0 m} \approx 80.4^{\circ}$.

By use of the data shown in table 6 and the in itial angle of simplified finite element model of QUY750 crawler crane boom, the angle of no.62 unit between the local coordinate system and global coordinate system is 77.4 degrees $\varphi_{p} \approx 77.4^{\circ}$ according to formula (24), which belongs to the simplified fin ite element model of QUY750 crawler crane boom. Thus the direction transformation matrix is (The design parameters of chord member cross-sectional area derive from axial force and axial stress. Due to the overlap between axial direction and $\mathrm{x}$ axis that is in the coordinate system, the row and column in transformation matrix corresponding to $u_{4}$ have been selected)

$$
T_{p p x}=\left[\begin{array}{llllll}
-0.2181 & -0.9759 & 0 & 0.2181 & 0.9759 & 0
\end{array}\right]
$$

On basis of the displacement similar relation, the angle of 1350tonnes crawler crane boom between the local coordinate system and global coordinate system is 72.3 degrees $\varphi_{p} \approx 72.3^{\circ}$. Direction transformation matrix, it would seem, is

$$
T_{p m x}=\left[\begin{array}{llllll}
-0.304 & -0.9527 & 0 & 0.304 & 0.9527 & 0
\end{array}\right]
$$

By figuring out magnification of boom that equals to 1.5625 accord ing to formu la no.25, the following matrices can be calculated.

$$
T_{\sigma p x}=\left[\begin{array}{llllll}
-0.1745 & -0.7807 & 0 & 0.1745 & 0.7807 & 0
\end{array}\right] \quad T_{\sigma p x}=\left[\begin{array}{llllll}
-0.2432- & 0.7622 & 0 & 0.2432 & 0
\end{array}\right] .
$$

On condition that the total cross-sectional area of chord member of QUY750 crawler crane boom is $A_{P}=50000 \mathrm{~mm}^{2}$. Using the formula no.29, the area of 1350 tonnes crawler crane boom is

$$
A_{m}=\frac{T_{p m x}}{T_{\sigma m x}} \frac{T_{p p x}^{T}}{T_{\sigma p x}^{T}} A_{P}=78122 \mathrm{~mm}^{2}
$$

For ensuring the security of similar design, the cross-sectional area of picked profile should be greater than calculated cross-sectional area slightly. Thus the stainless seamless steel pipe which type is $\phi 273 \times 26$ is selected in the paper.

\subsubsection{The similar design of truss boom for width and height}


The 1350tonnes crawler crane standard boom in width and height is designed in this paper based on the method which is defined in part 3.2. The selected parameters are shown in Tab. 7.

Table 7 Parameters of selection

\begin{tabular}{ccccc}
\hline & $\mu_{1}$ & $\mu_{2}$ & $\mu_{3}$ & $A_{1 x(y)}\left(\mathrm{mm}^{2}\right)$ \\
\hline Rotary plane & 1 & 1.04 & - & 4648 \\
Luffing plane & 2 & 1.04 & 0.7 & 5849.6 \\
\hline
\end{tabular}

According to formula no. 34 and the parameters above, the range of values for boom height and width is as following inequalities

$$
H \geq 2 \cdot \sqrt{\frac{l_{c x}^{2} \cdot A_{1 x}}{A_{1 x}[\lambda]^{2}-40 A}-\frac{I_{0}}{A_{0}}} \approx 2100 \mathrm{~mm} \quad B \geq 2 \cdot \sqrt{\frac{l_{c y}^{2} \cdot A_{1 y}}{A_{1 y}[\lambda]^{2}-40 A}-\frac{I_{0}}{A_{0}}} \approx 3000 \mathrm{~mm}
$$

In order to maintain the usability of similar design, the results above are rounded. Meanwhile the design results are extended for sake of safety.

\subsubsection{The similar design of truss boom for web member and the angle between chord member and web member}

On basis of discussion in part 3.3 and the boom framework of prototype QUY750, The similar design of 1350tonnes crawler crane truss boom for web member adopts the framework of type $\mathrm{W}$ which is welded in cross.

It has two kinds of standard section of QUY750 crawler crane boom, 6meters and 12meters. While the standard section length of large-tonnage crawler crane are generally 3 meters, 6meters and 12 meters. Thus the paper selected the 6 meters standard section for similar design.

On condition that the span of QUY750 standard section is $2596 \mathrm{~mm}$, so it can be calculated in similar design of 1350tonnes standard section by use of the formu la Eq. (35).

$$
c_{m}=n^{\prime} \cdot c_{p}=2596 m m
$$

Based on the lower limits of truss boom width and height, the angle between chord member and web member in luffing plane and rotary plane of $1350 t$ crawler crane is as following equations by use of Eq. (36).

$$
\left\{\begin{array}{l}
\alpha_{H}=90^{\circ}-\arctan \frac{c_{m}}{2 H}=90^{\circ}-\arctan \frac{n^{\prime} \cdot c_{p}}{2 H} \approx 53^{\circ} \\
\alpha_{B}=90^{\circ}-\arctan \frac{c_{m}}{2 B}=90^{\circ}-\arctan \frac{n^{\prime} \cdot c_{p}}{2 B} \approx 67^{\circ}
\end{array}\right.
$$

While the length of web me mber equals to

$$
\left\{\begin{array}{c}
l_{H}=H / \cos \left(90^{\circ}-\alpha_{H}\right)=2629.5 \mathrm{~mm} \\
l_{B}=B / \cos \left(90^{\circ}-\alpha_{B}\right)=3259.1 \mathrm{~mm}
\end{array}\right.
$$

\begin{tabular}{|c|c|c|c|c|}
\hline & & Design product & LR11350 & differences $(\%)$ \\
\hline \multirow{3}{*}{ Working condition } & Boom length $(m)$ & 150 & 150 & 0 \\
\hline & radius $(m)$ & 25 & 24 & 4.17 \\
\hline & load $(t)$ & 227.1 & 230.9 & 1.65 \\
\hline section & $\mathrm{W} \times \mathrm{H} \quad(\mathrm{mm})$ & $\begin{array}{l}\geq 3000 \\
\geq 2100\end{array}$ & $\begin{array}{l}3200 \\
2800\end{array}$ & - \\
\hline Chord member & type $(\mathrm{mm})$ & $\phi 273 \times 26$ & $\phi 298.5 \times 20$ & Smaller type \\
\hline \multirow{2}{*}{ Web member } & type $(\mathrm{mm})$ & $\phi 140 \times 7$ & $\phi 177.8 \times 8$ & Smaller type \\
\hline & luffing $(\mathrm{mm})$ & 2630 & 3027 & 13.12 \\
\hline
\end{tabular}

\subsection{The paramete is comparison of LR11350 and design product}

The parameters comparis on of LR1 1350 and design product is shown in Tab. 8 .

Table 8 Boom parameters comparison between design product and LR11350 


\begin{tabular}{|l|l|c|c|c|c|}
\hline & & revolving $(\mathrm{mm})$ & 3260 & 3400.4 & 4.13 \\
\cline { 2 - 6 } & \multirow{2}{*}{ angle } & luffing & $53^{\circ}$ & $68^{\circ}$ & 22 \\
\cline { 3 - 6 } & & revolving & $67^{\circ}$ & $70^{\circ}$ & 4.2 \\
\hline
\end{tabular}

From the Tab. 8, the dates suggest that the numerical errors in boom structure parameters between design product and LR11350 are less than 5\%, which derive from boom length, working radius, lifting load, web member length and the angle between chord member and web member.

The reasons why the chord member and web member of similar design have the smaller size compared to LR11350 are as follows:

(1) In the chord member selection, though outside diameter of design product is s maller than LR11350, but its wall thickness is greater than LR11350. Thus the cross-sectional area of single chord member is greater than LR11350 slightly.

(2) In terms of the web member selection, the paper uses the smaller size compared to it in prototype.

The reasons why the parameters of design product have large differences with LR1 1350 are as follows:

(1) The numerical error derives from the application of s mall deformation in local coordinate system, which is in comparison with actual deformation.

(2) As for the similar design in length of web member, the angle between chord member and web member, width and height of boom, the lower limits are applied to them.

(3) The prototype and LR11350 crawler crane have more safety margin .

\section{Conclusion}

For solving the lack of design type spectrum and rapid design method of large slenderness ratio truss boom, the works of this paper are as follows:

Based on the load combination II, (dead weight, lifting load, pulls of raising line and luffing line, wind load and inertia force under rotating and etc.) The mechanical models of crawler crane are established in luffing plane and rotary plane respectively.

In order to resolve the imperfection of classic similarity method applied in nonlinear field, the paper has established the nonlinear similar criterion by use of local coordinate system.

Select the QUY750 as prototype and wield the nonlinear similar criterion to rapidly design the 1350tonnes crawler crane. The parameters include cross-sectional area of chord member, the height and width of boom, the length of web member and the angle between chord member and web member. The comparison between design product and LR11350 is presented to prove the feasibility of the nonlinear similarity method.

\section{Acknowle dgments}

This work is supported by National Natural Science Foundation of China (No.51475068)

\section{References}

Cheng, H. Z. et al., Similarity criterion derivation and FEM verification of stress and strain fields of lithium-ion battery. Scientia Sinica Techologica. No.06 (2013), pp.670-683 (in Chinese).

Chetan, S. and Michelle, V., Virtual Manufacturing: an Overview. Computer and Engineering. Vol.31, No.02 (1996), pp.79-82.

Dong, S. L. et al., Research on geometrical nonlinear finite element method of spatial reticulated structures . Chinese Journal of Computational Mechanics. No.03 (2002), pp.365-368 (in Chinese).

Du, F. S., Theory of similarity finite element. Journal of Yanshan University. Vol.22, No.04 (1998), pp.286-289 (in 
Chinese).

Hida, M. and Okamoto, A., Development of automatic welding system for crawler crane latticed booms . Research and Development Kobe Steel Engineering Reports. Vol.57, No.01 (2007), pp.86-89.

Islam, K.M. A similarity coefficient measure and machine parts grouping in cellular manufacturing systems. International Journal of Production Research. Vo1.38, No.03 (2000).

Lei, Z. B., Dynamic similarity criteria for automotive structures of elastio-plastic materials under large deformation condition and precision of similarity calculation. Journal of Experimental Mechanics. Vol.15, No.04 (2000), pp.429-435 (in Chinese).

Lu, N. L. et al., Application of FEM of beam element with theory of II order. Journal of Harbin University of Civil Engineering and Architecture. No.04 (1998), pp.67-74 (in Chinese).

Mike, O., Manitowoc's new lattice-boom crawler crane. Diesel Progress North American Edition. Vol.64, No.09 (1998), pp.50-55.

Shi, M. J., Design of 250t crawler crane boom. Shandong University, (2010).

Standardization Admin istration of the People's Republic of China. Design rules for cranes, GB/T3811-2008[S]. (2008).

Sun, H. C. et al., Accurate algorith m for geometrically large deflection analysis of truss structures. Chinese Journal of Applied Mechanics. No.01 (2009), pp.45-50 (in Chinese).

Taesik, H., Similarity comparison of mechanical parts to reuse existing design. Computer Aided Designed. Vol.38 (2006).

Wang, H. H. and Shen, Z. Y., A practical method for dynamic analysis of plane-moving elastic beam-bar systems. Journal of Southeast University. No.S1 (1997), pp.97-104 (in Chinese).

Wang, H. H. et al., A simple method for geometric nonlinear analysis of plane beambar structures. Chinese Journal of Computational Mechanics. No.01 (1997), pp.121-125 (in Chinese).

Yuan, B. Z. Y. and Hu, S., The assembly model of hierarchical components. Journal of Computer-Aided Design\&Computer Graphics. Vol.12, No.06 (2000), pp.450-454 (in Chinese).

Zhang, H. H. and Lu, N. L., Large displacement analysis of tapered beam structures. Journal of Donghua University(English Edition). Vol.01, (2010), pp.117-122 (in Chinese). 